\title{
Catheter Ablation of Ventricular Tachycardia in Patients with Post-Infarction Cardiomyopathy
}

\author{
Babak Nazer, MD and Edward P Gerstenfeld, MD \\ Electrophysiology Section, Division of Cardiology, Department of Medicine, University of California San Francisco, San Francisco, CA, USA
}

Monomorphic ventricular tachycardia (VT) in patients with post-infarction cardiomyopathy (CMP) is caused by reentry through slowly conducting tissue with in areas of myocardial scar. The use of implantable cardioverter-defibrillators (ICDs) has helped to decrease the risk of arrhythmic death in patients with post-infarction CMP, but the symptomatic and psychological burden of ICD shocks remains significant. Experience with catheter ablation has progressed substantially in the past 20 years, and is now routinely used to treat patients with postinfarction CMP who experience VT or receive ICD therapy. Depending on the hemodynamic tolerance of $V T$, a variety of mapping techniques may be used to identify sites for catheter ablation, including activation and entrainment mapping for mappable VTs, or substrate mapping for unmappable VTs. In this review, we discuss the pathophysiology of VT in post-infarction CMP patients, and the contemporary practice of catheter ablation. (Korean Circ J 2014;44(4):210-217)

KEY WORDS: Tachycardia, ventricular; Catheter ablation; Myocardial infarction; Cardiomyopathy.

\section{Introduction}

Ventricular arrhythmias are a risk factor for sudden death among patients with post-infarction cardiomyopathy (CMP). ${ }^{122)}$ Sudden arrhythmic death from ventricular tachycardia (VT) or fibrillation is thought to account for approximately one-third of deaths in postinfarction CMP patients. ${ }^{344}$ Sustained monomorphic VT is typically caused by reentry though a heterogeneous infarct scar, and will be the focus of this review. Primary ${ }^{5-8)}$ and secondary ${ }^{9-11)}$ prevention trials have shown that implantable cardioverter-defibrillators (ICDs) decrease mortality among this patient population. However, ICD therapies-particularly shocks-are associated with depression and anxiety, ${ }_{1}^{12)}$ a decreased quality of life, ${ }^{12) 133}$ increased healthcare utilization ${ }^{14)}$ and mortality. ${ }^{15)}$ In ICD clinical trials, the incidence of appropri-

Correspondence: Edward P Gerstenfeld, MD, Electrophysiology Section, Division of Cardiology, Department of Medicine, University of California San Francisco, MU-East 4th Floor, 500 Parnassus Avenue, San Francisco, CA 94118, USA

Tel: 1-415-476-5706, Fax: 1-415-476-6260

E-mail: egerstenfeld@medicine.ucsf.edu

- The authors have no financial conflicts of interest.

This is an Open Access article distributed under the terms of the Creative Commons Attribution Non-Commercial License (http://creativecommons. org/licenses/by-nc/3.0) which permits unrestricted non-commercial use, distribution, and reproduction in any medium, provided the original work is properly cited. ate shocks for ventricular arrhythmias ranges from approximately $20 \%$ in primary prevention trials ${ }^{677}$ to $64 \%$ in the largest secondary prevention trial. ${ }^{9)}$ In the ALTITUDE registry of 185778 ICD patients, the incidence of appropriate shocks was $8 \%$ in the first year and $23 \%$ in five years. ${ }^{16)}$ Amiodarone, beta-blockers and sotalol have demonstrated modest efficacy in reducing shocks. However, in one randomized trial, the annual incidence of appropriate shocks in postinfarction CMP patients taking anti-arrhythmic medications remained at $6.7 \%{ }^{17)}$

Advances in catheter ablation and mapping technology have made catheter ablation an effective therapeutic option for patients with recurrent VT and appropriate ICD shocks. This article will discuss the pathophysiology of sustained monomorphic $\mathrm{VT}$ among patients with post-infarction CMP, and review the evidence and clinical practice of catheter ablation.

\section{Pathophysiology of Post-Infarction Ventricular Tachycardia}

Post-infarction VT is caused by reentry through diseased myocardium due to prior myocardial infarction. These areas of scar are comprised of fibrotic, unexcitable tissue, interspersed with areas of surviving, partially depolarizable myocytes (often referred to as "isthmuses" or "channels"), and areas of functional block that lead to slow conduction and unidirectional block critical to initiating reentry (Fig. 1). 
Scar sustaining post-infarction VT can be quite large, comprising up to $50 \%$ of the left ventricular (LV) surface area, ${ }^{18)}$ can contain several isthmuses and exit sites, and can lead to multiple VTs from the same scar area. Surface electrocardiogram (ECG) QRS morphology of a given monomorphic VT reflects only its exit site, and mapping with intracardiac electrograms (EGM) or three-dimensional (3D) electroanatomic systems is required to identify the extent of the scar and its associated isthmuses or channels. Early physiologic studies
A

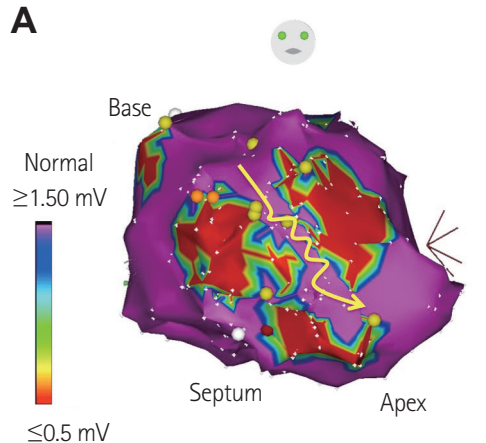

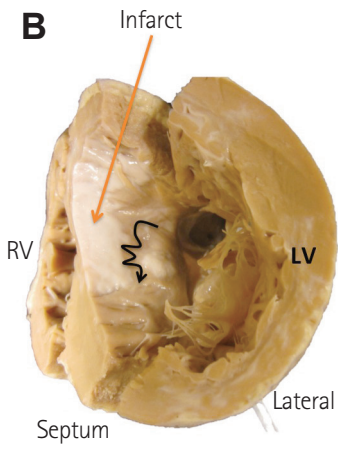

Dense scar

Fig. 1. Mechanism of macro reentry in scar-based VT. A: three-dimensional electroanatomic voltage map of LV with areas of normal myocardium (purple), scar (red), and border zone (other colors). The yellow arrow demonstrates a potential "isthmus" area between areas of scar that may harbor the VT circuit. B: autopsy specimen of a heart with prior myocardial infarction with a septal scar (pale area). Note that the scar is heterogeneous with areas of surviving myocardium between areas of infarcted myocardium that may serve as a potential VT isthmus (black arrow). This specimen is from a different patient than that of the electroanatomic map, and is used as an example. VT: ventricular tachycardia, LV: left ventricle, RV: right ventricle. found that most macro reentrant VTs have protected, narrow isthmuses that are required for maintenance, ${ }^{19220)}$ in 1990 Morady et al..$^{21)}$ first described the successful treatment of VT using radiofrequency (RF) catheter ablation in three patients.

\section{Indications for Catheter Ablation}

Patients with post-infarction CMP and ICDs who receive ICD shocks can be treated with either antiarrhythmic drugs or offered catheter ablation. In a patient without comorbidities who presents with ICD shocks for monomorphic VT, catheter ablation is a reasonable initial option at an experienced center, and may limit the longterm toxicities of antiarrhythmic drugs such as amiodarone. For patients already on antiarrhythmic drugs receiving ICD shocks, ablation is often the next best option. The European Heart Rhythm Association and Heart Rhythm Society have issued a joint expert consensus statement ${ }^{22)}$ with specific indications for ablation (Table 1).

Current RF ablation catheters are irrigated with saline to cool the catheter tip-tissue interface and allow the delivery of adequate power without char formation. ${ }^{23)}$ The first study of an irrigated catheter in patients with post-infarction VT demonstrated a 54\% freedom from VT at mean 243-day follow-up. ${ }^{24}$ Other trials of irrigated catheters have shown freedom from recurrent $V T$ in $51-53 \%$ of patients over 6-12-month follow-up. ${ }^{2526)}$ Catheter ablation has also been shown to be effective in patients with VT storm. ${ }^{27)}$

Previously, given the risk of undergoing an invasive procedure, catheter ablation of post-infarction VT was largely limited to

Table 1. Indications for catheter ablation

\section{Recommended for}

1. Symptomatic sustained monomorphic VT (SMVT), including VT terminated by an ICD, that recurs despite antiarrhythmic drug therapy or when antiarrhythmic drugs are not tolerated or not desired

2. Control of incessant SMVT or VT storm that is not due to a transient reversible cause

3. Frequent PVCs, NSVTs, or VT that is presumed to cause ventricular dysfunction

4. Bundle branch reentrant or interfascicular VTs

5. Recurrent sustained polymorphic VT and VF that is refractory to antiarrhythmic therapy when there is a suspected trigger that can be targeted for ablation Should be considered in

1. Patients who have one or more episodes of SMVT despite therapy with one of more Class I or III antiarrhythmic drugs

2. Patients with recurrent SMVT due to prior MI who have LV ejection fraction $>30 \%$ and expectation for 1 year of survival, and for whom it is an acceptable alternative to amiodarone therapy

3. Patients with hemodynamically tolerated SMVT due to prior MI who have a reasonably preserved LV ejection fraction (>35\%) even if they have not failed antiarrhythmic drug therapy

Contra-indicated

1. In the presence of a mobile ventricular thrombus (epicardial ablation may be considered)

2. For asymptomatic PVCs and/or NSVT that are not suspected of causing or contributing to ventricular dysfunction

3. For VT due to transient, reversible causes, such as acute ischemia, hyperkalaemia, or drug-induced torsade de pointes

VT: ventricular tachycardia, ICD: implantable cardioverter defibrillator, PVC: premature ventricular contraction, NSVT: non-sustained ventricular tachycardia, VF: ventricular fibrillation, MI: myocardial infarction, LV: left ventricle 
patients with recurrent ICD shocks. However, two randomized clinical trials tested the strategy of prophylactic VT ablation at the time of ICD implantation. In the VTACH study ${ }_{1}^{28)} 107$ patients with post-infarction CMP and stable VT were randomized to ICD versus ICD plus catheter ablation. At two years, $47 \%$ in the ablation group compared with 29\% in the control group were free of VT. The SMASH$V T$ study ${ }^{29)}$ also randomized patients who presented with VT (including unstable VT or VF) to ICD implantation with or without prophylactic VT ablation. Two-year freedom from VT was 88\% in the ablation group compared with 66\% in the control group. M-ore importantly, the frequency of future ICD shocks was lower in the ablation group ( $9 \%$ vs. 31\%; $p=0.003$ ). Neither study demonstrated a mortality benefit with prophylactic $\mathrm{VT}$ ablation, although there was a trend toward decreased mortality in the SMASH-VT study ( $9 \%$ vs. $17 \% ; p=0.29)$. Therefore, in some patients without significant comorbidities who initially present with monomorphic $V T$, prophylactic VT ablation at an experienced center can be considered prior to ICD placement.

\section{Pre-Procedural Considerations}

Post-infarction VT catheter ablation procedures are often complex and may involve repeated induction and prolonged mapping of sustained VT. Careful procedural planning, taking into consideration anesthesia requirements, comorbidities, anticoagulation, and reference ECG data, can help to ensure success and minimize com- plications.

When possible, 12-lead ECGs of the "clinical VT" (defined as the VT morphology occurring spontaneously) should be obtained. VT morphology helps to localize the exit site of the re-entrant circuit from the protected isthmus, and helps with procedural planning. A right bundle branch block morphology in lead V1 identifies a LV exit site, whereas left bundle branch block morphology suggests a $\mathrm{VT}$ exit in the right ventricle or, more commonly, LV septum. More specific localization can be achieved using leads AVR and AVL to distinguish septal from lateral exit, leads II, III, and AVF to distinguish superior from inferior exit, and the precordial leads to identify an apical/mid/basal exit (Fig. 2). Surface ECG localization allows for procedural planning, particularly in terms of vascular access, and for guiding the initial mapping procedure. In patients for whom surface ECG tracings of $\mathrm{VT}$ are not available, ICD interrogation can be helpful to define VT cycle length and intracardiac EGM morphology. After inducing VT during the ablation procedure, real-time ICD EGM morphology may be compared to the stored ICD EGM to determine if induced $\mathrm{VT}$ is, indeed, the clinical VT.

Myocardial ischemia can increase the risks of $\mathrm{VT}$ ablation and potentially contribute to the slow conduction that sustains $\mathrm{VT}$. It is, therefore, important to exclude myocardial ischemia with coronary angiography or a non-invasive stress test prior to any electrophysiology study. In the presence of ischemia, revascularization should be performed. Determining the location of any prior infarction using cardiac magnetic resonance imaging, nuclear imaging, or echocardiography
A

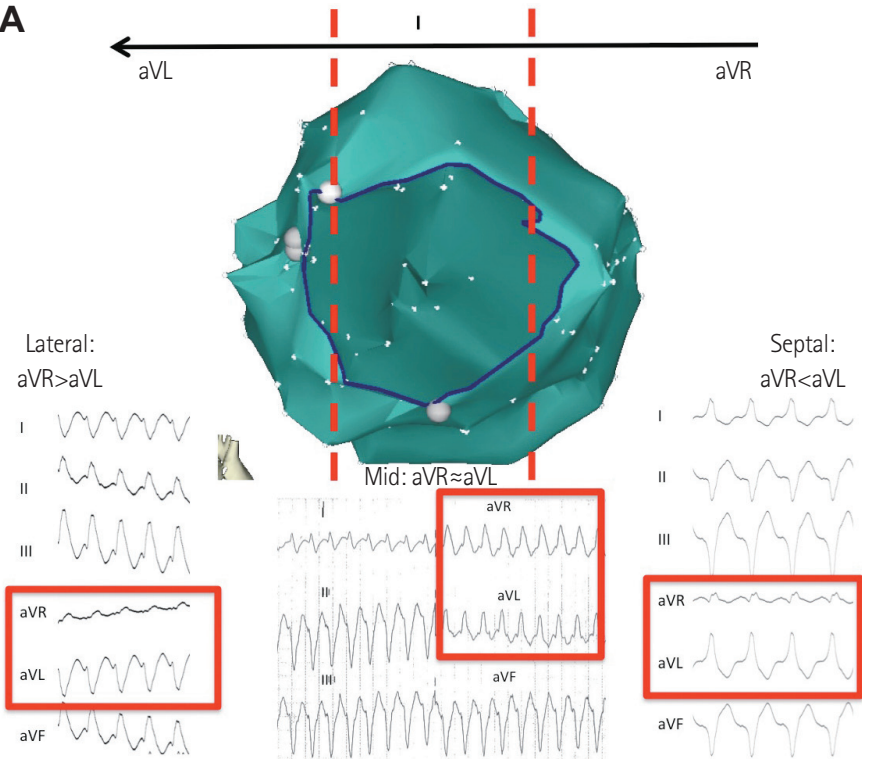

B

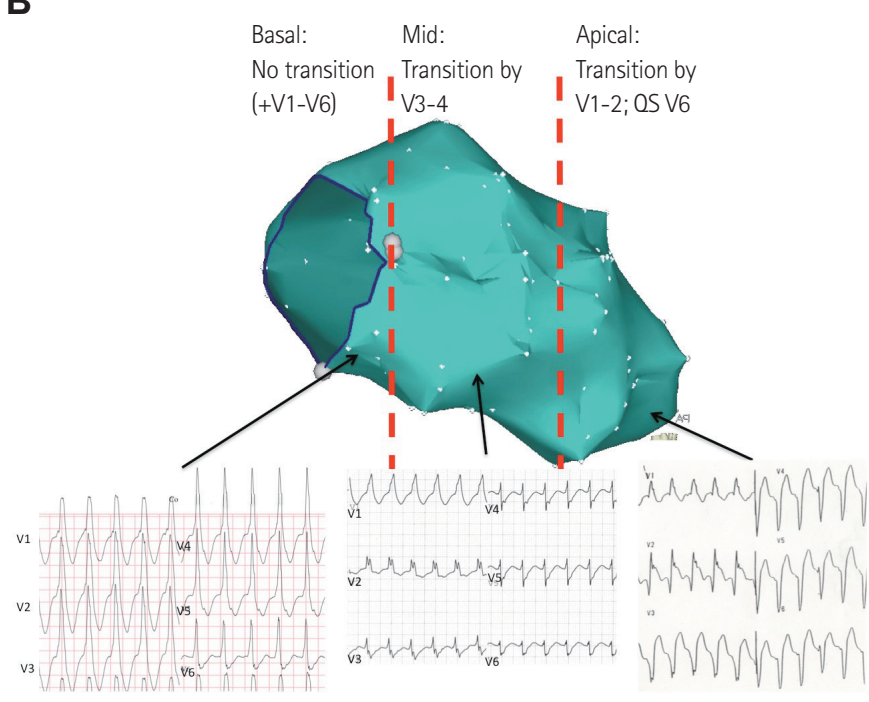

Fig. 2. Localization of $V T$ exit site using surface ECG. $A$ : for $V T s$ of septal origin, aVR is typically $<a V L$, while for lateral $V T s$ the opposite is true. From the mid-wall aVR=aVL. B: precordial leads are used to localize VTs from base to apex. Basal VTs are directed anteriorly towards the front of the body and therefore have positive precordial concordance. Mid-cavity VTs have RBBB morphology in V1 with a transition to a negative QRS at leads V3-4. Apical VTs have an early precordial transition by lead V3 and a OS pattern in V5-6. Combining the limb and precordial leads allows accurate localization of the VT exit site. VT: ventricular tachycardia, ECG: electrocardiogram, RBBB: right bundle branch block. 
is also helpful for guiding VT substrate mapping.

Antiarrhythmic medications should ideally be discontinued in advance of the procedure to maximize the chance of inducing VT. This may require hospital admission for with holding of antiarrhythmic medications in a safe environment. During mapping in the LV, anticoagulation is typically required to prevent thromboembolic phenomena, so consideration should be given to bleeding risks, particularly in patients on anti-platelet therapies. Post-procedurally, aspirin typically suffices, although a short course of warfarin may be considered when extensive LV ablation is performed and LV function is poor. Trans-thoracic echocardiography (preferably with contrast) should be performed to exclude mobile LV thrombus given the risk of embolization with catheter manipulation. However, the presence of laminated LV thrombus is not a formal contraindication. ${ }^{22)}$ Patients who also have persistent atrial fibrillation (AF) should receive four consecutive weeks of therapeutic anticoagulation or transesophageal echocardiography prior to their procedure, as defibrillation during $\mathrm{VT}$ ablation may lead to cardioversion of $A F_{\text {, and }}$ the associated risk of thromboemboli. Transesophageal echocardiography may also be useful in elderly patients to exclude significant aortic atherosclerosis. The LV may be accessed either via the femoral artery in retrograde fashion through the aorta and aortic valve, or via the femoral vein transeptally and across the mitral valve. Transseptal access should be used in patients with significant peripheral vascular or aortic valve disease.

Post-infarction CMP patients with severely reduced systolic function may require hemodynamic support to safely undergo VT ablation. Options include intra-aortic balloon pump, Impella micro-axial blood pump (Abiomed, Inc., Danvers, MA, USA), peripherallyinserted Tandem Heart (CardiacAssist, Inc., Pittsburg, PA, USA), or cardio-pulmonary bypass. No formal guidelines or data predict the need for mechanical support, but it may be considered in patients with severely reduced systolic function \{ejection fraction (EF) $<20 \%$ \} in whom spontaneous VT is not hemodynamically tolerated. The drawbacks of hemodynamic support are added procedural complexities and additional risks with large-bore arterial access-of particular concern among patients with significant peripheral vascular disease. Ultimately in these patients, substrate modification techniques (described below), which do not require the need for mapping during sustained VT, may be preferable to using these devices.

Patients with implanted left ventricular assist devices (LVAD) experience an incidence of ventricular arrhythmias as high as 52\% in one year. ${ }^{30)} \mathrm{VT}$ in LVAD patients may arise from the "suck-down" of the interventricular septum into the inflow cannula, a phenomenon more likely to occur in the setting of hypovolemia, and which must be excluded prior to the pharmacologic or catheter-based treatment of VT. Monomorphic VT in LVAD patients typically arises from pre-existing scar from the post-infarction CMP, or from reentry around the LV inflow cannula or its sutures. While sustained VT can be well-tolerated in patients with normally-functioning LVADs, ${ }^{31)}$ catheter ablation may be beneficial in LVAD patients who suffer from symptoms or right heart failure in the setting of $\mathrm{VT}^{32}{ }^{3233)}$

\section{Mapping and Ablation of Ventricular Tachycardia}

Sedation during VT procedures is often performed with the help of an anesthesiologist, and many centers prefer cardiac anesthesiology support for patients at high risk of hemodynamic deterioration. When possible, general anesthesia should be avoided as general anesthetics may suppress inducibility of $\mathrm{VT}$.

Typically, the first step in VT ablation procedures is to attempt to induce VT with paced ventricular extrastimuli. In a minority of patients-12\% of subjects in the VTACH tria ${ }^{28}-\mathrm{VT}$ is not inducible due to altered autonomic tone, antiarrhythmic medications, anesthesia or sedative medications. When VT is non-inducible, a limited "substrate modification" procedure may still be performed (as described below for "unmappable VT"). More commonly, even in patients who have one "clinical" VT morphology, multiple different VT morphologies can be induced. In the multicenter Thermocool trial, ${ }_{1}^{26)}$ a median three different VTs were inducible per patient. These different VT morphologies may arise from different areas of scar, or may simply represent different exit sites from the same scar. VTs that are hemodynamically unstable are typically referred to as "unmappable" VTs, whereas VTs that can be induced and sustained without hemodynamic compromise are considered "mappable." This distinction largely dictates the approach to VT mapping, as activation and entrainment mapping require stable sustained VT.

\section{Mappable ventricular tachycardia-activation and entrainment mapping}

If sustained $\mathrm{VT}$ is induced, an activation map can be created by the manipulation of the mapping catheter to record the earliest area of activation. An electroanatomic mapping system is useful for tracking activation in a 3D space. A mapping catheter is moved pointto-point around the ventricle, comparing the timing of each point's QRS onset to the reference fiducial point on a surface ECG lead. The 3D map is color-coded to highlight the area of earliest activation.

Entrainment mapping is then performed near the site of earliest activation. Briefly, entrainment mapping involves intermittently pacing near areas of scar at a rate slightly faster than the VT rate. The response to pacing and pattern of resumption of $V T$ after pacing help to identify the various components of the reentrant circuit (Fig. 3): entrance, isthmus, exit, inner loop, outer loop, adjacent bystander, or remote bystander. ${ }^{34)}$ 
After entrainment mapping, RF ablation is then typically performed focally at the critical isthmus site. This represents a narrow protected channel that is most likely to result in $\mathrm{VT}$ termination during ablation. These lesions may be extended to form a small $3-5 \mathrm{~cm}$ line to prevent VT recurrence. In some cases, ablation at an isthmus may "re-orient" the VT to take another path through the area of the scar to a different exit site with a slightly different morphology. Thus, entrainment mapping and isthmus ablation may need to be repeated over the same area of scar. It should be noted that after ablation of the mappable $\mathrm{V}$, many centers also perform additional substrate modification (discussed below).

\section{Unmappable ventricular tachycardia-voltage and pace mapping}

Approximately $70 \%$ of patients have hemodynamically unstable $\mathrm{VT}$ that precludes activation or entrainment mapping. ${ }^{26)}$ For these patients, voltage and pace mapping are used during sinus rhythm to identify optimal sites of RF ablation.

Voltage mapping utilizes electroanatomic mapping to create a 3D map of the ventricle, color-coded to the voltage of the intracardiac EGMs recorded at each point. For bipolar voltage maps, prior studies have shown that the fifth percentile of normal voltages in healthy hearts is $1.5 \mathrm{mV}$. Voltages $<0.5 \mathrm{mV}$ have been shown to correlate with dense scar. Therefore, color-coding of electroanatomic maps is typically standardized to depict voltage ranges of $>1.5 \mathrm{mV}$ (healthy or "purple"), $<0.5 \mathrm{mV}$ (scar or "red"), and border zone $(<1.5$

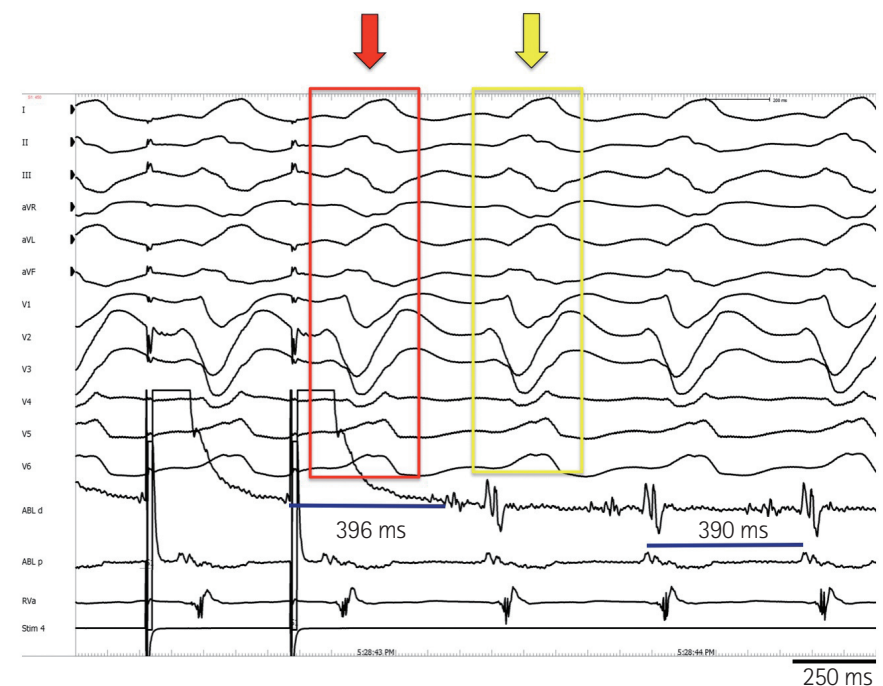

Fig. 3. Demonstration of concealed entrainment of a mappable VT. After induction of $V T$, pacing is performed from the suspected $V T$ exit site (orange arrow) at a rate 20-30 ms faster than the $V T$ rate (note pacing stimuli on the left side of the panel). Pacing is then stopped, and the post-pacing interval is compared to the VT cycle length; a difference $<30$ ms suggests that the pacing site is within the macroreentry circuit. The QRS morphology of the paced beat (red arrow and box) is identical to the spontaneous VT (yellow arrow and box) with a 12/12 lead match, identifying this site as a critical component of the VT circuit. VT: ventricular tachycardia.
$\mathrm{mV}$ and $>0.5 \mathrm{mV}$ ) (Fig. 4). These bipolar ranges reliably depict the location of prior infarction. Voltage ranges can also be altered to identify potential "channels" of relatively higher voltage within scar that may represent a critical isthmus for sustaining $\mathrm{VT}^{35)}$

When a 12-lead ECG of the clinical VT is available (either from patients' records or recorded after induction of a hemodynamically unstable $\mathrm{VT}$ at the start of the procedure), pace mapping can be performed by pacing from a catheter tip at various points near the suspected exit site of VT (often at the border zone of the scar identified

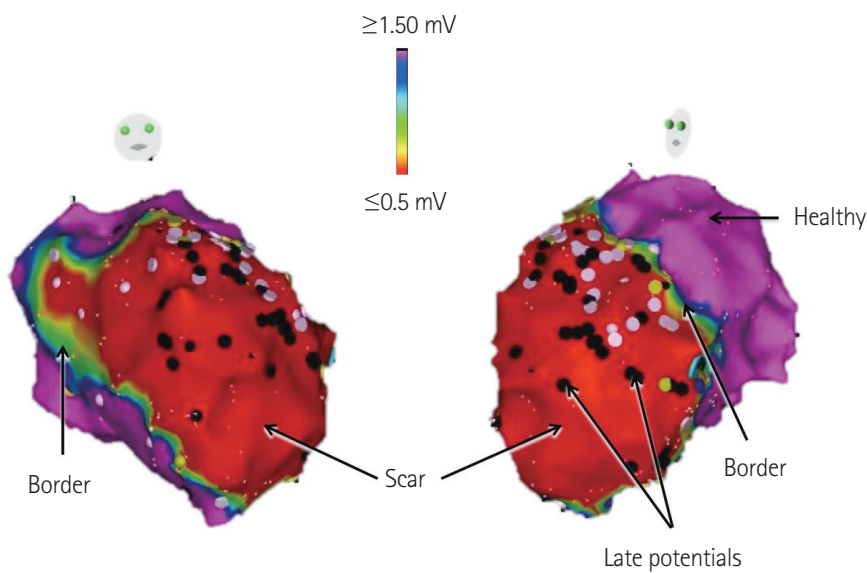

RAO

LAO

Fig. 4. Three-dimensional, electroanatomic voltage map in the right anterior oblique (RAO) and left anterior oblique (LAO) projections in a patient with a prior large anteroseptal myocardial infarction. Normal voltage is represented by regions with bipolar voltage $>1.5 \mathrm{mV}$ (purple), dense scar by regions with bipolar voltage $<0.5 \mathrm{mV}$, and scar border zone by regions with bipolar voltage $\geq 0.5$ and $\leq 1.5 \mathrm{mV}$. Black dots represent abnormal "late potentials" identified on intracardiac electrograms.

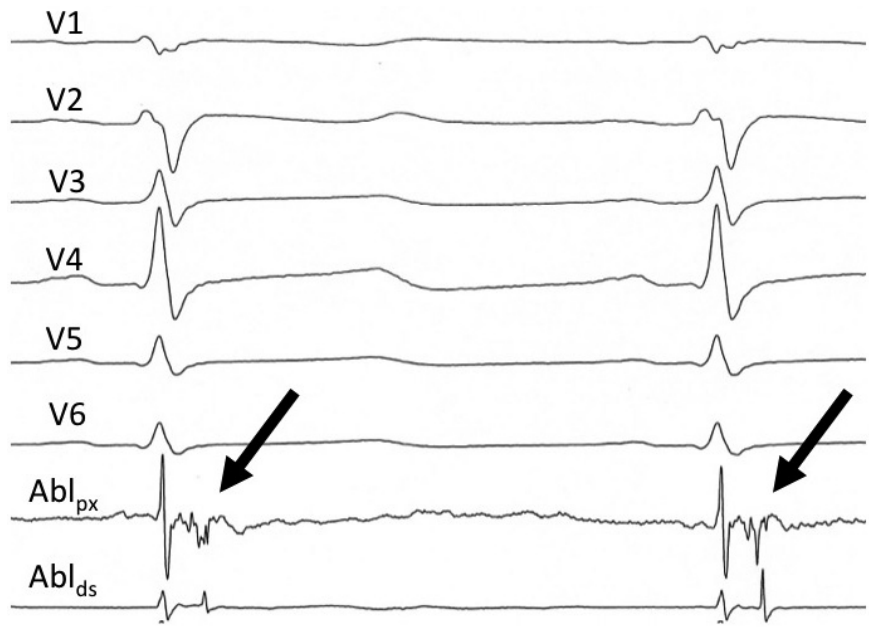

Fig. 5. Intracardiac electrogram demonstrating a high-frequency, low-amplitude late potential (solid arrows) after the QRS in sinus rhythm. This identifies a region of slow conduction that may be involved in VT maintenance, although such sites may also represent "bystanders" that are not important for VT maintenance. VT: ventricular tachycardia. 
by voltage map) until a pace map is identified that matches the morphology of the VT. RF ablation is then typically performed in a linear fashion through these matching pacemap regions in order to interrupt the presumed location of the VT circuit.

Intracardiac EGMs can also be scrutinized for low-amplitude, highfrequency signals that occur after the QRS complex, suggesting slow conduction (Fig. 5). Often called "late", "isolated", "delayed", or "diastolic" potentials, or "local abnormal ventricular activities", these potentials have been shown to correlate with infarct age ${ }_{1}^{36)}$ isthmus sites as identified by pace and entrainment mapping, ${ }^{37 / 38)}$ and channels as identified by voltage mapping. ${ }^{35)}$ Ablation of these potentials has been associated with a lower risk of recurrent VT in some series. $^{39440)}$

\section{Ablation Strategies and Procedural Endpoints}

Once optimal ablation sites are identified with the above mapping approach, irrigated RF energy is used to create lesions at powers ranging from 30-50 W. With externally irrigated catheters, attention should also be paid to patients' volume status, as saline infusion rates range $8-15 \mathrm{~mL} / \mathrm{min}$ during ablation, and patients often receive over one liter of fluid.

It should be noted that there are no studies comparing the efficacy of the aforementioned approaches to mapping; most centers use a combination. Typically, after ablation of mapped sites, re-induction of VT is repeated with ventricular extrastimuli. Procedural endpoints used to define "successful ablation" include: 1) non-inducibility of the clinical VT, 2) non-inducibility of all VTs, and 3) elimination of all late potentials. In the Multicenter Thermocool trial, inducibility of any $\mathrm{VT}$ at the end of the procedure was associated with almost double the rate of $V T$ recurrence, although there was no increased risk when the analysis was limited to inducible VTs that were faster than the clinical VT. ${ }^{26)}$ Finally, an approach of empiric "scar homogenization" with endocardial and epicardial ablation of all abnormal potentials was shown to decrease VT recurrence when compared to standard endocardial mapping and ablation alone in one single center retrospective study, ${ }_{1}^{41)}$ and this requires further investigation. Ultimately, the choice of the endpoint must be chosen based on procedural time and the patient's clinical status, as prolonged, repeated attempts at $\mathrm{VT}$ induction, pacing, and ablation may lead to adverse outcomes in the clinically brittle post-infarction CMP population. If stimulation is not performed at the end of a procedure due to hemodynamic instability, non-invasive programmed stimulation (NIPS) via the ICD can be performed one to two days later. Induction of clinic VT during NIPS correlates with a high likelihood of recurrent $\mathrm{VT}^{42)}$

Epicardial ablation, while often necessary for non-ischemic CMP
$V T$, is not routinely performed in post-infarction $V T$, since scar related to myocardial infarction is predominantly sub-endocardial. However, after unsuccessful endocardial ablation, epicardial mapping and ablation may be considered in post infarction CMP patients who have not undergone prior cardiac surgery (as prior surgery causes adhesions which increase the risk of subxyphoid epicardial access). Epicardial VT circuits are identified in approximately 10\% of postinfarction VT studies, and are more often associated with prior inferior wall infarcts. ${ }^{43}$

\section{Complications}

Multi-center studies of post-infarction VT ablation have demonstrated approximately $8 \%$ of major complication rates related to $\mathrm{VT}$ ablation, which is most often due to incessant $\mathrm{VT}^{\left.24{ }^{2} 26\right)}$ The most common complication is vascular injury (pseudoaneurysm, arteriovenous fistula, dissection), particularly with arterial access and retrograde aortic access of the left ventricle, occurring in approximately $2 \%$ of procedures. ${ }^{2224-27)}$ Stroke or transient ischemic attack occurs in 1-2\%, highlighting the need for judicious anticoagulation with LV mapping and ablation. Cardiac perforation and tamponade can occur in $1 \%{ }^{24-27)}$ In patients with septal VTs, ablation near the His bundle can lead to heart block. ${ }^{24)}$ Heart failure exacerbations may occur after post-infarction VT ablation, ${ }_{1}{ }^{6)}$ and may be due to irrigation volume infused or "stunning" effects from numerous RF lesions. The SMASH-VT trial, however, routinely monitored LV EF before and after $V T$ ablation, and found no difference between preablation EF and EF at three and 12 months post-ablation. ${ }^{29)}$ Peri-procedural mortality ranges from 0 to $3 \%$ in major clinical trials, ${ }^{2426) 28229)}$ and was $1.75 \%$ in a recent registry-based study. ${ }^{44)}$

\section{Emerging Technologies}

Remote magnetic navigation ("Stereotaxis") techniques have gradually expanded to electrophysiology procedures, and have demonstrated some efficacy in post-infarction VT. ${ }^{45}$ Remote navigation offers the hope of reducing fluoroscopy and procedure times. While remote navigation can acquire detailed LV substrate maps, modest contact force with the LV endocardium may limit the depth of delivered ablation lesions.

Scar in the inter-ventricular septum often presents a challenge for endocardial ablation, as the protected VT circuit can be located deep in the septum, and may not be approachable from the right or LV endocardium or epicardium. In this setting, a cooperative effort between electrophysiologists and interventional cardiologists may be required to ablate the $\mathrm{VT}$ with transcoronary ethanol infusion. ${ }^{46)}$ Other approaches for deep septal VTs include bipolar ablation on 
both sides of the septum, ${ }^{47)}$ or use of a saline-infused RF needle ablation catheter. ${ }^{48)}$

\section{Summary}

Post-infarction VT represents a significant source of mortality and symptoms, particularly among patients with ICDs; antiarrhythmic medications such as amiodarone provide modest efficacy with significant side-effects and toxicities. RF catheter ablation of post-infarction VT results in approximately 50-75\% freedom of VT after one year, as well as a significant reduction in the frequency of ICD shocks. A variety of mapping and ablation approaches may be used depending on inducibility and hemodynamic tolerance of $\mathrm{VT}$, targeting either critical isthmuses of macro-reentrant loop, or late potentials. Procedural risks can be reduced by careful planning and patient selection.

\section{Acknowledgments}

The authors would like to thank Dr. Philip Ursell for his assistance with photographs of pathology specimens.

\section{References}

1. Mukharji J, Rude RE, Poole WK, et al. Risk factors for sudden death after acute myocardial infarction: two-year follow-up. Am J Cardiol 1984; 54:31-6.

2. Anderson KP, DeCamilla J, Moss AJ. Clinical significance of ventricular tachycardia (3 beats or longer) detected during ambulatory monitoring after myocardial infarction. Circulation 1978;57:890-7.

3. Greenberg H, Case RB, Moss AJ, et al. Analysis of mortality events in the Multicenter Automatic Defibrillator Implantation Trial (MADIT-II). J Am Coll Cardiol 2004;43:1459-65.

4. Narang R, Cleland JG, Erhardt L, et al. Mode of death in chronic heart failure. A request and proposition for more accurate classification. Eur Heart J 1996;17:1390-403.

5. Buxton AE, Lee KL, Fisher JD, Josephson ME, Prystowsky EN, Hafley G. A randomized study of the prevention of sudden death in patients with coronary artery disease. Multicenter Unsustained Tachycardia Trial Investigators. N Engl J Med 1999;341:1882-90.

6. Bardy GH, Lee KL, Mark DB, et al. Amiodarone or an implantable cardioverter-defibrillator for congestive heart failure. N Engl J Med 2005; 352:225-37.

7. Moss AJ, Zareba W, Hall WJ, et al. Prophylactic implantation of a defibrillator in patients with myocardial infarction and reduced ejection fraction. N Engl J Med 2002;346:877-83.

8. Moss AJ, Hall WJ, Cannom DS, et al. Improved survival with an implanted defibrillator in patients with coronary disease at high risk for ventricular arrhythmia. Multicenter Automatic Defibrillator Implantation Trial Investigators. N Engl J Med 1996;335:1933-40.

9. A comparison of antiarrhythmic-drug therapy with implantable defi- brillators in patients resuscitated from near-fatal ventricular arrhythmias. The Antiarrhythmics versus Implantable Defibrillators (AVID) Investigators. N Engl J Med 1997;337:1576-83.

10. Connolly SJ, Gent M, Roberts RS, et al. Canadian implantable defibrillator study (CIDS) : a randomized trial of the implantable cardioverter defibrillator against amiodarone. Circulation 2000;101:1297-302.

11. Kuck KH, Cappato R, Siebels J, Rüppel R. Randomized comparison of antiarrhythmic drug therapy with implantable defibrillators in patients resuscitated from cardiac arrest: the Cardiac Arrest Study Hamburg (CASH). Circulation 2000;102:748-54.

12. Hegel MT, Griegel LE, Black C, Goulden L, Ozahowski T. Anxiety and depression in patients receiving implanted cardioverter-defibrillators: a longitudinal investigation. Int J Psychiatry Med 1997;27:57-69.

13. Schron EB, Exner DV, Yao 0, et al. Quality of life in the antiarrhythmics versus implantable defibrillators trial: impact of therapy and influence of adverse symptoms and defibrillator shocks. Circulation 2002;105: 589-94.

14. Moss $A J$, Greenberg $H$, Case RB, et al. Long-term clinical course of patients after termination of ventricular tachyarrhythmia by an implanted defibrillator. Circulation 2004;110:3760-5.

15. Sweeney MO, Sherfesee L, DeGroot PJ, Wathen MS, Wilkoff BL. Differences in effects of electrical therapy type for ventricular arrhythmias on mortality in implantable cardioverter-defibrillator patients. Heart Rhythm 2010;7:353-60.

16. Saxon $L A$, Hayes DL, Gilliam FR, et al. Long-term outcome after ICD and CRT implantation and influence of remote device follow-up: the ALTITUDE survival study. Circulation 2010;122:2359-67.

17. Connolly SJ, Dorian P, Roberts RS, et al. Comparison of beta-blockers, amiodarone plus beta-blockers, or sotalol for prevention of shocks from implantable cardioverter defibrillators: the OPTIC Study: a randomized trial. JAMA 2006;295:165-71.

18. Haqqani HM, Kalman JM, Roberts-Thomson KC, et al. Fundamental differences in electrophysiologic and electroanatomic substrate between ischemic cardiomyopathy patients with and without clinical ventricular tachycardia. J Am Coll Cardio/ 2009;54:166-73.

19. Okumura K, Olshansky B, Henthorn RW, Epstein AE, Plumb VJ, Waldo $\mathrm{AL}$. Demonstration of the presence of slow conduction during sustained ventricular tachycardia in man: use of transient entrainment of the tachycardia. Circulation 1987;75:369-78.

20. de Bakker JM, van Capelle FJ, Janse MJ, et al. Reentry as a cause of ventricular tachycardia in patients with chronic ischemic heart disease: electrophysiologic and anatomic correlation. Circulation 1988;77: 589-606.

21. Morady F, Frank $R$, Kou WH, et al. Identification and catheter ablation of a zone of slow conduction in the reentrant circuit of ventricular tachycardia in humans. J Am Coll Cardiol 1988;11:775-82.

22. Aliot EM, Stevenson WG, Almendral-Garrote JM, et al. EHRA/HRS Expert Consensus on Catheter Ablation of Ventricular Arrhythmias: developed in a partnership with the European Heart Rhythm Association (EHRA), a Registered Branch of the European Society of Cardiology (ESC), and the Heart Rhythm Society (HRS); in collaboration with the American College of Cardiology (ACC) and the American Heart Association (AHA). Europace 2009;11:771-817. 
23. Ruffy R, Imran MA, Santel DJ, Wharton JM. Radiofrequency delivery through a cooled catheter tip allows the creation of larger endomyocardial lesions in the ovine heart. J Cardiovasc Electrophysiol 1995;6: 1089-96.

24. Calkins $H$, Epstein $A$, Packer $D$, et al. Catheter ablation of ventricular tachycardia in patients with structural heart disease using cooled radiofrequency energy: results of a prospective multicenter study. Cooled RF Multi Center Investigators Group. J Am Coll Cardiol 2000;35:190514.

25. Tanner $H_{1}$ Hindricks $G$, Volkmer $M$, et al. Catheter ablation of recurrent scar-related ventricular tachycardia using electroanatomical mapping and irrigated ablation technology: results of the prospective multicenter Euro-VT-study. J Cardiovasc Electrophysiol 2010;21:47-53.

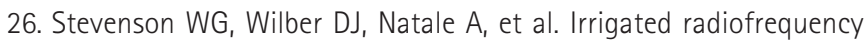
catheter ablation guided by electroanatomic mapping for recurrent ventricular tachycardia after myocardial infarction: the multicenter thermocool ventricular tachycardia ablation trial. Circulation 2008; 118:2773-82.

27. Carbucicchio C, Santamaria M, Trevisi N, et al. Catheter ablation for the treatment of electrical storm in patients with implantable cardioverterdefibrillators: short- and long-term outcomes in a prospective single-center study. Circulation 2008;117:462-9.

28. Kuck KH, Schaumann A, Eckardt $L$, et al. Catheter ablation of stable ventricular tachycardia before defibrillator implantation in patients with coronary heart disease (VTACH): a multicentre randomised controlled trial. Lancet 2010;375:31-40.

29. Reddy VY, Reynolds MR, Neuzil P, et al. Prophylactic catheter ablation for the prevention of defibrillator therapy. N Engl J Med 2007;357: 2657-65.

30. Andersen M, Videbaek R, Boesgaard S, Sander K, Hansen PB, Gustafsson $F$. Incidence of ventricular arrhythmias in patients on long-term support with a continuous-flow assist device (HeartMate II). J Heart Lung Transplant 2009;28:733-5.

31. Oz MC, Rose EA, Slater J, Kuiper JJ, Catanese KA, Levin HR. Malignant ventricular arrhythmias are well tolerated in patients receiving longterm left ventricular assist devices. J Am Coll Cardiol 1994;24:1688-91.

32. Osaki $S$, Alberte $C$, Murray MA, et al. Successful radiofrequency ablation therapy for intractable ventricular tachycardia with a ventricular assist device. J Heart Lung Transplant 2008;27:353-6.

33. Dandamudi G, Ghumman WS, Das MK, Miller JM. Endocardial catheter ablation of ventricular tachycardia in patients with ventricular assist devices. Heart Rhythm 2007;4:1165-9.

34. Stevenson $W G$, Khan $H$, Sager $P$, et al. Identification of reentry circuit sites during catheter mapping and radiofrequency ablation of ventricular tachycardia late after myocardial infarction. Circulation 1993; 88(4 Pt 1):1647-70.

35. Mountantonakis SE, Park RE, Frankel DS, et al. Relationship between voltage map "channels" and the location of critical isthmus sites in pa- tients with post-infarction cardiomyopathy and ventricular tachycardia. J Am Coll Cardiol 2013;61:2088-95.

36. Bogun $F$, Krishnan $S$, Siddiqui $M$, et al. Electrogram characteristics in postinfarction ventricular tachycardia: effect of infarct age. J Am Coll Cardiol 2005;46:667-74.

37. Bogun $F_{1}$ Good E, Reich $S$, et al. Isolated potentials during sinus rhythm and pace-mapping within scars as guides for ablation of post-infarction ventricular tachycardia. J Am Coll Cardio/ 2006;47:2013-9.

38. Hsia HH, Lin D, Sauer WH, Callans DJ, Marchlinski FE. Relationship of late potentials to the ventricular tachycardia circuit defined by entrainment. J Interv Card Electrophysio/ 2009;26:21-9.

39. Arenal A, Glez-Torrecilla E, Ortiz M, et al. Ablation of electrograms with an isolated, delayed component as treatment of unmappable monomorphic ventricular tachycardias in patients with structural heart disease. J Am Coll Cardio/ 2003;41:81-92.

40. Jaïs $P$, Maury $P$, Khairy $P$, et al. Elimination of local abnormal ventricular activities: a new end point for substrate modification in patients with scar-related ventricular tachycardia. Circulation 2012;125:218496.

41. Di Biase L, Santangeli P, Burkhardt DJ, et al. Endo-epicardial homogenization of the scar versus limited substrate ablation for the treatment of electrical storms in patients with ischemic cardiomyopathy. J Am Coll Cardio/ 2012;60:132-41.

42. Frankel DS, Mountantonakis SE, Zado ES, et al. Noninvasive programmed ventricular stimulation early after ventricular tachycardia ablation to predict risk of late recurrence. J Am Coll Cardio/ 2012;59: 1529-35.

43. Verma A, Marrouche NF, Schweikert RA, et al. Relationship between successful ablation sites and the scar border zone defined by substrate mapping for ventricular tachycardia post-myocardial infarction. J Cardiovasc Electrophysiol 2005;16:465-71.

44. Harikrishnan $P_{\text {, Kolte }}$, Palaniswamy $C_{1}$ et al. Catheter ablation of ventricular tachycardia: ten-year trends in utilization, in-hospital complications, and in-hospital mortality in patients with ischemic cardiomyopathy. J Am Coll Cardiology 2014;63(12 Suppl 1):A294. Abstract.

45. Aryana $A$, d'Avila $A$, Heist EK, et al. Remote magnetic navigation to guide endocardial and epicardial catheter mapping of scar-related ventricular tachycardia. Circulation 2007;115:1191-200.

46. Sacher F, Sobieszczyk P, Tedrow U, et al. Transcoronary ethanol ventricular tachycardia ablation in the modern electrophysiology era. Heart Rhythm 2008;5:62-8.

47. Koruth JS, Dukkipati S, Miller MA, Neuzil P, d'Avila A, Reddy VY. Bipolar irrigated radiofrequency ablation: a therapeutic option for refractory intramural atrial and ventricular tachycardia circuits. Heart Rhythm 2012;9:1932-41.

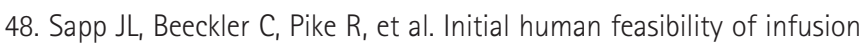
needle catheter ablation for refractory ventricular tachycardia. Circulation 2013;128:2289-95. 